\title{
EVALUACIÓN DE TRATAMIENTOS PARA LA PROPAGACIÓN VEGETATIVA DE TRIPEPERRO (Philodendron longirrhizum M. M. Mora \& Croat), ESPECIE DE USO ARTESANAL EN EL DEPARTAMENTO DEL QUINDÍO.
}

\author{
TREATMENT EVALUATION FOR VEGETATIVE PROPAGATION OF TRIPEPERRO \\ (Philodendron longirrhizum M. M. Mora \& Croat) \\ HANDCRAFTS-USED SPECIE IN QUINDIO DEPARTMENT.
}

\author{
Luz Adriana Gómez-Marín ${ }^{1}$, Ana Lucia Lopez-gonzalez ${ }^{2}$
}

Fecha de recibido: Diciembre 21 de 2010

Fecha de Aceptado: Julio 12 de 2011

Correspondencia: Centro de Estudios e Investigaciones en Biodiversidad - CIBUQ. Universidad del Quindío. Av. Bolivar Calle 12 Norte Armenia Quindío Colombia.

Licenciatura en Biología y Educación Ambiental - Universidad del Quindío. E-mail: adju21@yahoo.es

Docente programa de Biología. Directora CIBUQ. Universidad del Quindío. E-mail: analucia@uniquindio.edu.co

\section{RESUMEN}

El Tripeperro (Philodendron longirrhizum) es una especie de amplio uso para la producción de artesanías en el Departamento del Quindío; motivo por el cual se evaluó su propagación vegetativa como alternativa para minimizar el impacto negativo de su explotación. En la finca La Aldana de la Universidad del Quindío, se sembraron estacas bajo nueve tratamientos, consistentes en la adición de fertilizante orgánico (lombricompuesto) y fertilizante químico (25-5-10 NPK), con o sin la aplicación directa de un enraizador a base de Ácido Naftalén Acético (ANA). Los tratamientos se realizaron bajo un diseño de bloques aleatorizados, cada uno con 10 repeticiones, para un total de 90 estacas evaluadas; realizándose seguimiento quincenal durante nueve meses. Se estableció que los tratamientos con fertilizante Químico y el control, donde no se aplicó ningún tipo de sustancia, produjeron mejores resultados en cuanto al desarrollo de las yemas foliares, el número promedio de hojas y el ancho promedio de la lámina foliar. A partir de los resultados se plantea que la propagación de Philodendron longirrhizum por vía vegetativa es viable y de bajo costo para el campesino, puesto que se puede realizar sembrando en tierra de capote sin adicionar ningún fertilizante; por tanto, se puede afirmar que a través de esta estrategia se puede contribuir a la conservación de la especie y garantizar la continuidad de la actividad cestera en el Quindío.

Palabras clave: Philodendron longirrhizum, tripeperro, propagación vegetativa.

\begin{abstract}
The "tripeperro" (Philodendron longirrhizum) is a widely used species for the production of handicrafts in the Department of Quindío. That is why vegetative propagation was evaluated as an alternative to minimize the negative impact of their exploitation. In the farm called "La Aldana", property of the University of Quindio, stem cuttings were planted in nine treatments consisting of the addition of organic fertilizer (vermicompost) and chemical fertilizer (NPK 25-5-10) with or without the direct application of a rooting to base of naphthalene acetic acid (NAA). The treatments were conducted under randomized block design, each with 10 repetitions, for a total of 90 stem cuttings evaluated, carried out fortnightly monitoring for nine months. It was established that chemical fertilizer treatments and control, which did not apply any type of substance, produced better results in the development of leaf buds, the average number of leaves and the average width of the leaf blade. From the results, it is suggested that the propagation of Philodendron longirrhizum by a vegetative way is viable and low cost to the farmer since it can be planted in soil without adding any fertilizer, so it can be said that this strategy may contribute to the conservation of the species and ensure continuity in the baskets and handicrafts business in the department of Quindío.
\end{abstract}

Key words: stem cuttings, "tripeperro", vegetative propagation. 


\section{INTRODUCCIÓN}

La actividad artesanal está íntimamente ligada a la memoria tradicional de los pueblos haciendo parte de su cultura material y comercial (1). Es así como en el Eje Cafetero se realiza la labor de la cestería, la cual requiere de materias primas extraídas del medio natural de lo que en general se denominan bejucos, entre los cuales están especies como Smilax tomentosa (atacorral), Asplundia sarmentosa (cestillo), Chusquea latifolia (chusco) y Philodendron longirrhizum (tripeperro) (2).

El agotamiento de las poblaciones locales de bejucos repercute en dificultades económicas para los artesanos, obligándolos a cambiar sus actividades tradicionales por otras más rentables, con la consecuente pérdida de identidad cultural (2).

A pesar de la importancia que tiene la actividad cestera como fuente generadora de empleo y por ende, de ingresos económicos de los campesinos; se dedica poca atención por parte de entidades competentes, a la realización de estudios que contribuyan a cuantificar y disminuir el grado de presión al que están siendo sometidos los bejucos; además, es poco lo que se ha estudiado sobre los aspectos biológicos de las especies trabajadas, lo que ha impedido formular propuestas para su conservación en las regiones donde son aprovechadas (3).

Es innegable que en el caso de las plantas silvestres que son explotadas para esta actividad, las tasas de extracción son tan altas que no son sostenibles y llevan al agotamiento local de las poblaciones (4). Se ha estimado que un artesano extrae en promedio alrededor de 82 libras de tripeperro verde con cáscara al mes, equivalente a 25 libras seco sin cáscara, suficiente para elaborar 125 artesanías de 100 g (2). Esta situación no sólo amenaza la misma producción artesanal, sino que genera un detrimento de la flora nativa del país, con la consecuente pérdida de la biodiversidad.

La actividad artesanal consume básicamente recursos naturales como fuentes de materias primas, siendo la tejeduría (57.52\%) y la madera (13.48\%) los oficios más sobresalientes. La actividad cestera en el norte del Quindío no cubre los costos en los que se incurre para producir las artesanías; se estiman los costos totales para 1997 en $\$ 105.125 .775$ y los ingresos totales por venta en $\$ 41.186 .397$ (4) por lo que desde este punto de vista la actividad no es rentable y a esto debería sumarse el impacto sobre las fuentes de fibras vegetales, lo que probablemente se refleja en que el $45.35 \%$ de la población sufre de escasez de materia prima y el $29.94 \%$ tiene dificultades en cuanto a la calidad de la misma (5).

Aunque los artesanos colectores saben de la disminución de algunas especies de uso artesanal, conocen requisitos para la colección del material y reconocen estrategias de conservación para las especies con que trabajan; en su mayoría no aplican medidas que tiendan a conservarlas debido, entre otros factores, a la presión ejercida por la demanda de productos y a que no son dueños de las tierras donde hacen la extracción (6).

En el caso de plantas explotadas en exceso, como las de uso artesanal, el cultivo puede representar una solución viable para obtener el material necesario, sin poner en peligro la supervivencia de las especies y garantizando la obtención de materia prima (4). Es necesario ofrecer a los artesanos colectores alternativas viables para la conservación de las especies vegetales con las cuales trabajan, por tanto, se requiere conocer aspectos como tratamientos que garanticen el éxito en la propagación vegetativa de las plantas, el modo de siembra más eficiente, el tiempo normal de brote y el estado general de las plántulas en un tiempo determinado, de tal manera que el mismo artesano pueda cultivar las especies que utiliza, contribuyendo así a la conservación de las plantas y de la actividad cestera.

Por lo anterior y con el fin de estudiar una alternativa para minimizar el impacto negativo de la explotación del tripeperro, se evaluó una estrategia para su propagación vegetativa en condiciones de vivero y bajo diferentes tratamientos, con el fin de establecer una propuesta para el sector campesino que deriva su sustento de fibras naturales.

\section{MATERIALES Y MÉTODOS}

Área de estudio: Los ensayos de propagación se desarrollaron en la Finca La Aldana de la Universidad del Quindío, ubicada en predios del relicto vegetal "Cedro Rosado", a una altitud de 1520 m (7). 
Trabajo de campo: Se recolectaron estacas de Philodendron longirrhizum en el relicto vegetal de la finca la Soledad, en el municipio de Filandia, Quindío, ubicado entre los $04^{\circ} 40^{\prime} 29^{\prime \prime} \mathrm{N}$ y $74^{\circ} 39^{\prime} 44^{\prime \prime} \mathrm{W}$, a una altitud de $1800 \mathrm{~m}$, (8). Es de anotar que la recolección se realizó en temporada de invierno. Para la recolección de estacas se tuvo en cuenta que las plantas parentales presentaran tallos con un diámetro entre 3 y $6 \mathrm{~cm}$., se cortaron en forma de bisel 90 estacas ( 10 para cada uno de los tratamientos) con una longitud entre 17 y $22 \mathrm{~cm}$, teniendo en cuenta la presencia de por lo menos 2 yemas por estaca.

Las estacas se sembraron en bolsas de polietileno de
$1.5 \mathrm{~K}$ que fueron llenadas con tierra de capote. Cada grupo de 10 estacas fue sometido a un tratamiento diferente (Tabla 1), utilizando enraizador Hormonagro \#1 (acido1-Naftalenacético) en dos concentraciones (0.5 g y $0.1 \mathrm{~g})$, el fertilizante químico 25-5-10 NPK diluido en agua $(70 \mathrm{~g} / \mathrm{L})$ y el fertilizante orgánico Lombricompuesto (287.9 g adicionado directamente en las bolsas).

El enraizador fue aplicado una sola vez en el momento de la siembra y los fertilizantes químico y orgánico se agregaron dos veces en el transcurso del experimento (una al mes de sembradas las estacas y la segunda a los 5 meses de siembra).

Tabla 1. Tratamientos evaluados en la propagación vegetativa de Philodendron longirrhizum.

\begin{tabular}{cccc}
\hline & $\begin{array}{c}\text { Fertilizante } \\
\text { Orgánico }\end{array}$ & Fertilizante Químico & Control sin fertilizante \\
\hline Enraizador 0.5 g & Tratamiento 1 & Tratamiento 2 & Tratamiento 3 \\
Enraizador $\mathbf{0 . 1} \mathbf{g}$ & Tratamiento 4 & Tratamiento 5 & Tratamiento 6 \\
$\begin{array}{c}\text { Control sin } \\
\text { enraizador }\end{array}$ & Tratamiento 7 & Tratamiento 8 & Tratamiento 9* \\
\hline
\end{tabular}

Las bolsas se ubicaron en una zona de sombrío en la orilla del relicto vegetal Cedro Rosado. Cada 15 días y durante un periodo de 9 meses, a las 90 estacas sembradas se les midió la sobrevivencia, el número de nuevas yemas foliares, el número promedio de hojas establecidas; el tiempo promedio de aparición de hojas y las medidas del ancho y la longitud de la lámina foliar.

\section{Procesamiento de Datos}

Se realizó un análisis de bloques completos aleatorizados con 10 repeticiones por tratamiento. Los datos de número promedio de nuevas yemas foliares en la estaca inicial y el número promedio de hojas se trasformaron con base en la fórmula $\sqrt{x+0.5}$. Para evaluar la existencia de homogenidad de varianza y/o distribución normal de los datos. Se realizó un test para la homogeneidad de varianza de Cochran C, Hartley, Bartlett (9) y para la prueba de Normalidad un test Shapiro- Wilk. Los datos obtenidos se sometieron a análisis de varianza, en los casos en que se presentaron diferencias estadísticamente significativas se realizó la prueba LSD de comparación de medias con el fin de establecer entre qué promedios se presentaron diferencias estadísticamente significativas. Esto cálculos fueron realizados con el software Statistix 8.0 y las gráficas realizadas con Statistica 5.1.

\section{RESULTADOSY DISCUSION}

\section{Eficiencia de los tratamientos}

Porcentaje de sobrevivencia: El tratamiento 9 (control) presentó el mayor porcentaje de sobrevivencia con 92.08\%, seguido por el tratamiento 7 (fertilizante orgánico) con una sobrevivencia de $86.2 \%$ y el tratamiento 4 compuesto por enraizador en concentración $0.1 \mathrm{~g}$ y fertilizante orgánico con $80.4 \%$. El tratamiento 3 (enraizador en concentración de $0.5 \mathrm{~g}$ ) presentó la menor sobrevivencia con $59.8 \%$ (Figura. 1.) 


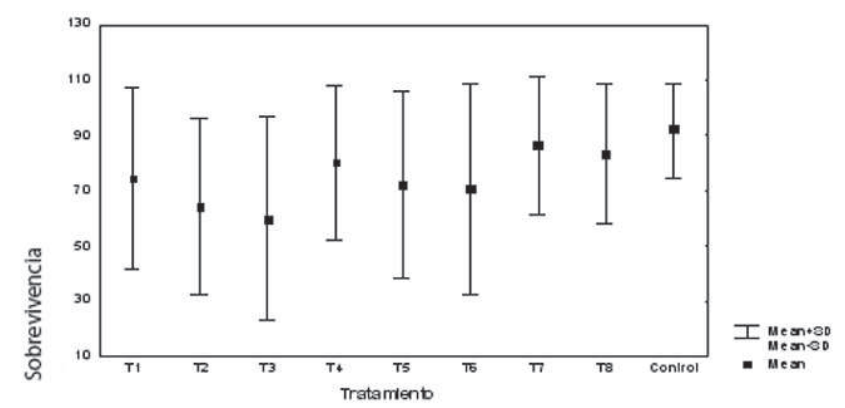

Figura 1. Porcentaje de sobrevivencia de las estacas de Philodendron longirrhizum, entre los tratamientos evaluados.

Las pequeñas variaciones entre el porcentaje de sobrevivencia para cada uno de los tratamientos fueron corroboradas con el análisis estadístico, el cual indicó que no hay diferencias estadísticamente significativas en esta variable para los nueve tratamientos.

No se encontraron diferencias significativas en el porcentaje de sobrevivencia de las estacas entre tratamientos (ANOVA $\mathrm{P}=0.4615 ; \mathrm{F}=0.98 ; \mathrm{gl}=89$ datos trasformados con base en $\operatorname{Arcsen} \sqrt{x}$. Este tipo de resultados pueden ser inducidos por diversas situaciones, una de estas es que las estacas de tripeperro presentan un alto contenido de reservas y la presencia de una corteza protectora adecuada para conservar la humedad (10).

Estas condiciones (acumulación de reservas y humedad) sumada a la ausencia de hojas y raíces, hacen que las estacas reduzcan su tasa de metabolismo y que por tanto, no obtengan ni pierdan cantidades considerables de estas sustancias, lo que puede favorecer su sobrevivencia.

\section{Crecimiento y desarrollo}

Número promedio de nuevas yemas foliares formadas en la estaca inicial: El tratamiento que obtuvo mayor número de yemas fue el 8 (fertilizante químico 25-5-10 NPK), en el cual se formaron en promedio 1.5 yemas por estaca (Figura. 2). Por el contrario, el tratamiento 3 (enraizador en concentración de $0.5 \mathrm{gr}$ ) presentó el promedio más bajo (0.3) en la emisión de estas estructuras. Se encontraron diferencias significativas en cuanto al número de nuevas yemas producidas por las estacas entre tratamientos (ANOVA P $=0.0119$; $\mathrm{F}=2.69$; $\mathrm{gl}=89$ - datos trasformados con base en $\sqrt{x+0.5}$-); es decir, puede asumirse que hubo un efecto de los tratamientos sobre la emisión de nuevas yemas. La prueba LSD indicó que el tratamiento 8 presentó el mayor promedio (1.5).

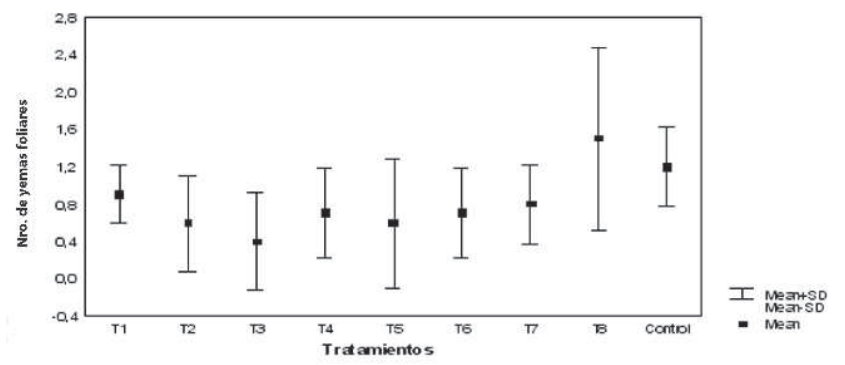

Figura 2. Distribución del número promedio de nuevas yemas foliares en la estaca inicial de Philodendron longirrhizum, entre los tratamientos evaluados.

La diferencia estadísticamente significativa entre el número promedio de nuevas yemas foliares en la estaca inicial, con relación al tratamiento conformado por fertilizante químico, muestra la acción de los minerales $\mathrm{N}$, P y K sobre el rompimiento de la latencia y la estimulación para la diferenciación de yemas, lo cual coincide con los planteamientos de Montoya (11) quien afirmó que la presencia de Potasio y Fósforo en sustratos favorecen el desarrollo y brote vigoroso de yemas en reposo.

Adicionalmente, según este mismo autor, debe considerarse que en las plantas, la regulación de la expresión de los eventos fisiológicos está en función de la cantidad y tipo de hormonas presentes, es decir, depende del balance hormonal, por lo que en este caso también es posible que las hormonas endógenas presentes en las estacas pudieron influenciar los resultados obtenidos y haber sido potenciadas por la adición del fertilizante.

De acuerdo a esto, la propagación vegetativa de Philodendron longirrhizum, parece estar relacionada directamente con el potencial que tienen las estacas de esta especie para producir yemas y lograr que estas se desarrollen, dado que lo esencial para la propagación de una especie por vía vegetativa es la capacidad para la producción y activación de yemas lo cual garantiza que se pueda dar un adecuado desarrollo de la planta (12).

Número promedio de hojas: La cuantificación de esta variable se hizo a partir de las yemas que se desarrollaron, generando ramas y hojas. El tratamiento 9 (control) presentó el mayor promedio de hojas (4.3), mientras que el tratamiento 1 (enraizador en concentración de $0.5 \mathrm{~g}$ y fertilizante orgánico) presentó el menor número promedio (0.2) de hojas (Figura. 3). 
Se encontraron diferencias significativas en el número de hojas emitidas por las estacas entre tratamientos (ANOVA $\mathrm{P}=0.0083 ; \mathrm{F}=2.85 ; \mathrm{gl}=89$ - datos trasformados con base en $\sqrt{x+0.5}$-), es decir, hubo efecto de los tratamientos sobre el número de hojas emitidas por las estacas. La prueba LSD indicó que las estacas sometidas al tratamiento 9 presentaron el mayor promedio (4.3) en la emisión de hojas, en contraste con el tratamiento 1 que presentó el menor promedio (0.2) en el número de hojas emitidas.

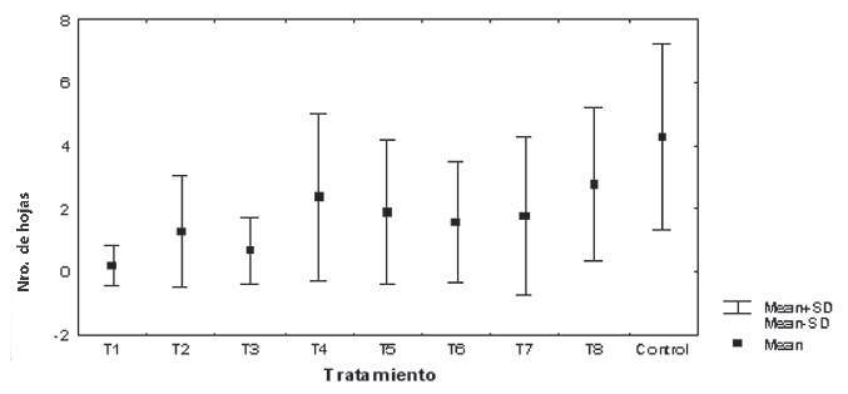

Figura 3. Número promedio de hojas de estacas de Philodendron longirrhizum, entre los tratamevaluados.

La eficiencia en cuanto al número de hojas de las estacas establecidas bajo el tratamiento 9 (control) puede explicarse por las características de los suelos del trópico por que presentan un horizonte $A$ conocido como capote rico en materia orgánica con una amplia gama de macro y micronutrientes en diferentes concentraciones, que favorecen el desarrollo de la vegetación que en ellos crece (13). En este sentido, la siembra en tierra de capote resulta siendo, por sí mismo, un tratamiento eficiente para la propagación vegetativa de la especie evaluada y guarda relación con que las plantas poseen hormonas endógenas que se encuentran en concentraciones variables (14) y que para el caso del tripeperro, son suficientes para generar una respuesta positiva en el desarrollo.

En relación al crecimiento y desarrollo de las hojas en la propagación vegetativa de Philodendron longirrhizum, se encontró que el tratamiento 8 (fertilizante Químico 25-5-10 NPK) y el tratamiento 9 (control), presentaron el mayor ancho de la hoja con un promedio de (5.8 y 16 $\mathrm{cm}$ respectivamente); mientras que los otros tratamientos formaron un grupo homogéneo (Figura. 4).

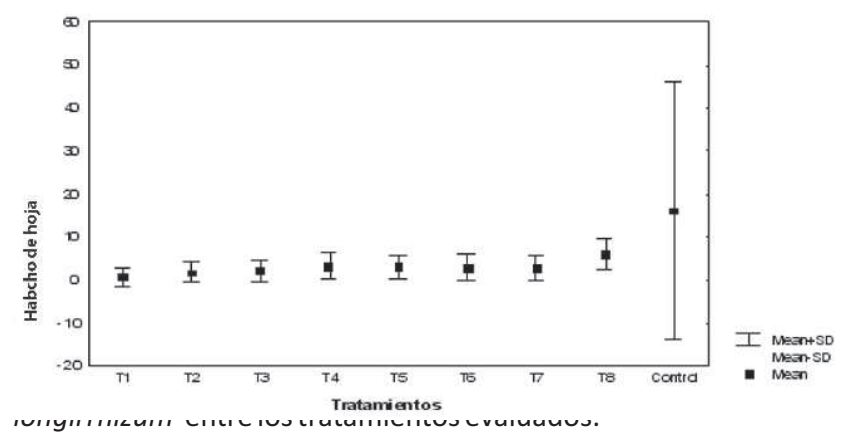

Se encontró diferencias significativas en el promedio del ancho de las hojas emitidas por las estacas entre tratamientos (ANOVA $\mathrm{P}=0.0008 ; \mathrm{F}=4.95 ; \mathrm{gl}=38$ homogenización de los datos), es decir, hubo efecto de los tratamientos sobre el ancho de la hoja de Philodendron longirrhizum. Este resultado fue confirmado por la prueba LSD.

La diferencia encontrada en cuanto al ancho de las hojas indica que esta variable tiende a relacionarse con la presencia de nutrientes en el sustrato y también podría considerarse como efecto de la presencia de hormonas endógenas como las auxinas y citoquininas, que por intervenir en la expansión y alargamiento celular de las hojas, generan efecto sobre la variación en el ancho de la lámina foliar como fue reportado por Ansorena (15).

Con relación al análisis estadístico de la longitud de las hojas entre tratamientos no se encontró diferencias significativas entre los tratamientos (ANOVA $P=$ 0.1866; $\mathrm{F}=1.56 ; \mathrm{gl}=38$ ), es decir, los tratamientos no influyeron diferencialmente sobre la longitud de la hoja de Philodendron longirrhizum. (Figura 5)

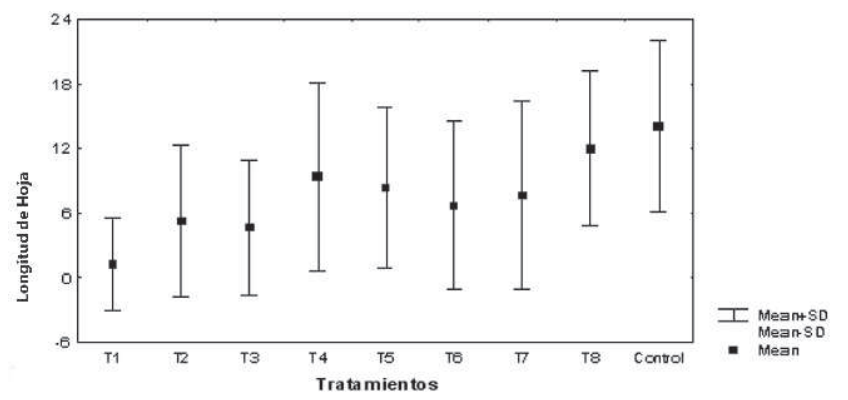

Figura 5. Promedio de la longitud de las hojas en estacas Philodendron longirrhizum, entre los tratamientos evaluados.

Este resultado puede asumirse como la confirmación de que la división y expansión celular son dos 
fenómenos del crecimiento controlados genéticamente, quizás en parte a través de la actividad de las sustancias del crecimiento y de la herencia (16).

Día de aparición de las hojas: No se encontraron diferencias significativas entre los tratamientos en cuanto al tiempo promedio de aparición de las hojas (ANOVA P $=0.6270 ; \mathrm{F}=0.78$; gl = 38). Este resultado puede explicarse por los ritmos internos que tiene cada especie, por lo que las estacas que fueron cortadas y sembradas el mismo día tienden a presentar la activación de yemas y su posterior diferenciación en intervalos de tiempo similares (Figura 6).

Finalmente, puede decirse que los tratamientos 8 y 9 , fueron los más eficientes en cuanto al mayor número de nuevas yemas foliares, mayor promedio de hojas y mayor ancho promedio de la lámina foliar, datos que fueron confirmados por la prueba LSD. De esta manera se puede establecer que la mejor forma de propagar Philodendron longirrhizum, por vía vegetativa, es la siembra de estacas en tierra capote con o sin combinación de un fertilizante químico que contenga macronutrientes (NPK) en proporciones de 25-5-10. La proporcionalidad de nutrientes aporta al desarrollo del brote foliar y por ende a la sobrevivencia y desarrollo de la estaca, toda vez que los procesos de formación y desarrollo de las hojas son fundamentales y tienden a estar sincronizados con el desarrollo de la planta, dado el rol de estas estructuras en la fotosíntesis (17).

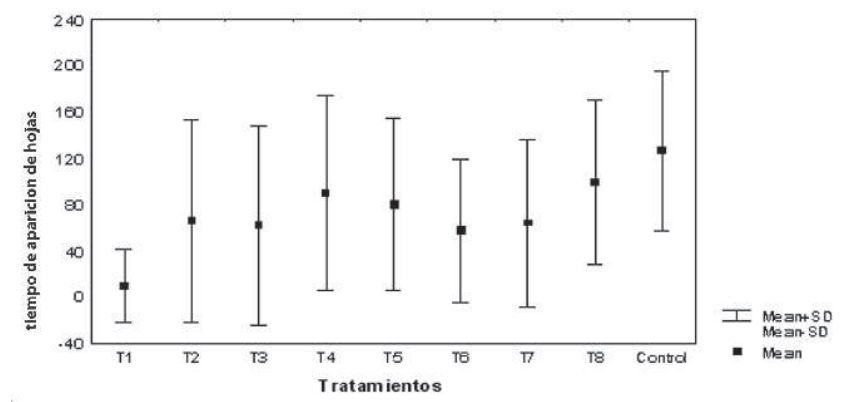

Figura 6. Tiempo promedio del día de aparición de las hojas en estacas Philodendron longirrhizum, entre los tratamientos evaluados.

Considerando que si se desea multiplicar una especie, la propagación vegetativa representa una enorme ventaja, desde el punto de vista económico y biológico (18), El éxito obtenido en la propagación vegetativa de Philodendron longirrhizum justifica la utilización de esta técnica con fines de contribuir a la conservación de las poblaciones naturales de esta especie, por tratarse de una alternativa de bajo costo y alta efectividad.

\section{CONCLUSIONES}

$\checkmark$ La propagación vegetativa es una técnica viable y de bajo costo para producir plantas de tripeperro

(Philodendron longirrhizum), por lo que se convierte en una opción práctica para garantizar la producción de un elevado número de plántulas que contribuyan a aumentar el número de individuos en las poblaciones naturales de esta especie, a través de reforestaciones programadas.

$\checkmark$ Los tratamientos que generaron los mejores resultados en la propagación vegetativa de Philodendron longirrhizum, con relación al mayor número de nuevas yemas foliares en la estaca inicial, mayor número promedio de hojas y mayor ancho promedio de la lámina foliar, fueron los tratamientos de siembra en tierra de capote con la adición de fertilizante Químico y siembra en tierra de capote sin añadir ninguna sustancia; lo que permite seleccionar al segundo tratamiento debido a que los campesinos pueden utilizarlo sin incurrir en gastos económicos, se genera menor impacto en el suelo donde se cultiven y por tanto, su implementación puede conllevar a un uso sustentable de la materia prima.

\section{AGRADECIMIENTOS}

Este proyecto fue posible gracias al apoyo logístico y del personal del Centro de Estudios e Investigaciones en Biodiversidad y Biotecnología de la Universidad del Quindío CIBUQ. 


\section{BIBLIOGRAFIA}

(1) Zapata, R. (2001). Instalación de un Jardín Clonal de Especies de Interés Artesanal en el Departamento del Quindío, Trabajo de Grado Licencado en Biología y Educación Ambiental. Universidad del Quindío Facultad de Ciencias Básicas y Tecnologías.

(2) Ramos, B. (1997) Estrategias para el manejo sostenible y uso artesanal de tripeperro Philodendron hastatum, en los departamentos de Caldas, Quindío y Risaralda. Cali: Convenio FES - Artesanías de Colombia e Instituto de Investigaciones Alexander Von Humboldt. .

(3) ALDANA, J. (2005) .Marco metodológico para desarrollar proyectos piloto de manejo de especies de flora y fauna silvestre. Bogotá: Informe presentado al Instituto Alexander von Humboldt.

(4) Ramos, B. (2001) Hacia un uso sostenible de las materias primas silvestres utilizadas en artesanías. Metodología de evaluación de propuesta de buen uso. Cali: Convenio FES- Artesanías de Colombia e Instituto de Investigaciones Alexander Von Humboldt.

(5) Artesanías de colombia S.A, 1998.Ministerio de Desarrollo Económico. Censo Económico Nacional del sector artesanal. Santa fé de Bogotá.

(6) Lopéz, A. (2006). Sistemática y Propagación In Vitro de Tripeperro (Philodendron sagittifolium), Especie de Uso Artesanal Amenazada en el Quindío. Armenia: Centro de Investigaciones en Biodiversidad.

(7) Castro, G. (1995). Inventario de Flora del Relicto Vegetal y Elaboración de Folletos para los visitantes al Sendero de Interpretación Ambiental Cedro Rosado de la Universidad del Quindío. Armenia: Trabajo de Grado (licenciado en Biología y Educación Ambiental).

(8) MARTíneZ, y. y ÁlVAREZ, L. (2006). Diseño e implementación de un plan de co-manejo adaptativo de bejucos utilizados en artesanías en la Zona Cafetera colombiana. Bogotá: Informe presentado al Instituto Alexander von Humboldt

(9) Cochran, C, Hartley, W. y Bartlett, G. (1947) "Some Consequences When the Assumptions for the Analysis of Variance are not Satisfied" Biometrics. New York.

(10) Segura, A, Martinez, R, Ariz, F, Argel, G. y Treviño, T. (1991). propagacion Agamica de seis Especies Forestales Neotropicales en Colombia. Bogotá: En CONIIF.

(11) Montoya, H. (1994). Fisiología de la Reproducción Vegetal. Medellín: Universidad Nacional de Colombia.

(12) Buitrago, Cy Romero, F. (2001) Suelos Agrícolas. Mexico: Editorial Revolucionaria.

(13) Hugo, R y Saldivar, L. (1994). Fisiología Vegetal. Editorial Trillas.

(14) Hernández, J. Aramendi, H y Cardona, C. (2005). “Influencia del acído indolbutirico y acidonaftalenoacetivo sobre el enraizamiento esquejes de caña flecha (Gynerium sagittatum Aubl.)" Revista facultad de ciencias Agricolas.Universidad de Cordoba.(10), 5-13.

(15) Ansorena, M. (1994). Sustratos: Propiedades y Caracterización. Madrid: Editorial Mundi-Prensa. 
16. ROST, T, Botero, C. y Hernandez, A. (1992). Botánica "Introducción a la Biología Vegetal". Mexico:Editorial Limusa.

(17) Svenson, S. y Davies, F. (1995) Change in tissue mineral elemental concentration during root initiation and development of poisenttia cuttings. Texas: Aandm University, college Station,

(18) Rojas, G. (1993). Fisiología Vegetal Aplicada. Editorial Interamericana Mc Graw Hill. 4 edición. 